\title{
MÉDIATION ET ANGLICISMES EN FRANÇAIS ET EN ITALIEN
}

\author{
Michele DE GIOIA \\ Université de Padoue
}

\begin{abstract}
En): As a result of the harmonizing framework of European law, mediation practices in France and Italy are highly influenced by North American concepts. Legally, mediation is reduced to one of the modes of conflict resolution aiming to avoid recourse to institutional justice. Linguistically, discourse on mediation is increasingly full of anglicisms, observable, for instance, on official Web sites or in a terminographic bilingual book. This article provides illustrations of this influence from a comparative French-Italian terminological perspective.

Résumé (Fr) : Sous l'effet du cadre harmonisateur du droit européen, les pratiques de la médiation en France et en Italie subissent l'influence de la conception nord-américaine. Sur le plan juridique, la médiation se réduit à un des modes de règlement des conflits visant à éviter le recours à la justice institutionnelle. Sur le plan linguistique, les discours sur la médiation sont de plus en plus émaillés d'anglicismes, observables entre autres sur des sites Web officiels ainsi que dans un ouvrage terminographique bilingue. Cette étude illustre cette influence dans une perspective terminologique comparée français-italien.
\end{abstract}

Keywords (En): Mediation; anglicisms; French-Italian comparative terminology

Mots-clés (Fr) : Médiation ; anglicismes ; terminologie comparée français-italien

\section{Présentation}

Cette étude s'insère dans le cadre d'un projet de recherche de l'Université de Padoue visant la construction d'une ontoterminologie de la médiation ${ }^{1}$. Pour ce faire, il est nécessaire de bien cerner la définition de médiation vu l'influence conceptuelle et linguistique du modèle nord-américain. Cet article analyse cette influence dans une perspective terminologique comparée français-italien et s'inscrit ainsi en linguistique appliquée, définie par HUMBLEY (2017 : 135) citant Linn, comme «la recherche de solutions à des problèmes - généralement de communication - de la vie réelle, par le biais de la théorie et de l'analyse linguistiques, et cela dans une démarche nécessairement pluridisciplinaire (LINN, 2011)».

\section{L'influence nord-américaine conceptuelle et linguistique}

Sous l'effet du cadre harmonisateur du droit européen, les pratiques de la médiation en France et en Italie subissent l'influence de la conception nordaméricaine. Celle-ci range la médiation sous l'étiquette d'Alternative Dispute Resolution $(A D R)$ qui s'applique également à la négociation, à la conciliation et aux autres modes alternatifs servant à résoudre les litiges en dehors du cadre judiciaire traditionnel. Cette conception est imprégnée de «la forte propension procédurière

\footnotetext{
${ }^{1}$ Projet BIRD161093/16 de l'Université de Padoue. Nous remercions Mario Marcon pour ses suggestions pertinentes.
} 
des Américains »(GuILlaume-HofNung, $2015: 12$ ) et se reflète, entre autres, dans le développement de certaines formations universitaires. En guise d'exemple, citons le rayonnement du cursus de négociation et de médiation tenu par l'Harvard Program of Negotiation. L'ouvrage issu de ce cursus, intitulé Getting to yes: negotiating agreement without giving in (FISHER, URY \& PATTON, 1982), continue de jouer un rôle central pour la formation non seulement des négociateurs, mais aussi des médiateurs dans le monde entier ${ }^{2}$. Nous remarquons la superposition, voire l'interchangeabilité, linguistique et conceptuelle, entre médiation et négociation. Néanmoins, tout en partageant des caractéristiques communes comme leur nature privée et le caractère volontaire des participants, ces deux modalités se distinguent - du moins, du point de vue théorique - l'un(e) de l'autre. D'une part, la négociation peut se passer d'un tiers, alors que ce dernier est indispensable pour la médiation. D'autre part, la première prévoit un compromis que la seconde essaie plutôt de dépasser (GUILLAUME-HOFNUNG, $2015: 80$ ).

Quand on parle de modèle nord-américain procédurier de la médiation, on ne saurait oublier la contribution canadienne ${ }^{3}$ «qui reste pour certains courants européens la référence absolue » (Ibidem: 13). De l'expérience canadienne, nous retenons surtout la relation de subordination causale de la médiation à la conciliation. En s'appuyant sur la loi 65 de 1997, modifiant le Code de procédure civile québécois en matière de médiation familiale, la médiation se transforme en outil indispensable pour atteindre la conciliation (ou la réconciliation) entre les parties (Ibidem: 14). C'est surtout «l'impasse de la médiation-conciliation » (Ibidem : 14) qui se répand en Europe par l'intermédiaire du Canada.

Depuis les années 1980-1990, des réflexions scientifiques sur la médiation se développent en France et mettent en relief l'attention à l'altérité. SIX (1990) ${ }^{4}$ considère cet intérêt comme un moyen de responsabiliser l'individu vis-à-vis de l'autre et de la paix sociale. La médiation met de côté tout souci procédurier et remet au centre l'être humain, l'égalité de tous les êtres humains, la reconnaissance de l'Autre et la responsabilité citoyenne. En Italie, parmi de rares réflexions autochtones sur ce sujet se distingue l'ouvrage de CASTELLI (1996) qui se nourrit par ailleurs de certaines références françaises.

Par rapport au modèle nord-américain se développe un modèle français de la médiation qui se situe au sein de la communication éthique et de l'éthique de la communication. Ce modèle souligne surtout l'autonomie du processus - non pas de la procédure - de la médiation par rapport au conflit. Nous nous référons à la définition formulée par GUILLAUME-HOFNUNG (2015 : 70) :

\footnotetext{
${ }^{2}$ Le rayonnement international de cet ouvrage est également attesté par ses traductions en français (Comment réussir une négociation. Nouvelle édition suivie des réponses aux questions des lecteurs, $3^{\mathrm{e}}$ éd. française, Paris, Seuil, 2006) et en italien (L'arte del negoziato, Milan, Corbaccio, [2005]).

${ }^{3}$ Bonafé-Schmitt (2013) parle de modèles «anglo-saxons » en ne se focalisant que sur les ÉtatsUnis.

${ }^{4}$ Six est souvent considéré comme le père de la médiation, mais il faut rappeler qu'à partir des années 80, en France, quand on parlait de médiation on se référait à trois penseurs et pionniers des pratiques médiatives, à savoir Jean-François Six lui-même, Michèle Guillaume-Hofnung et Régine Langendorff ; on les appelait souvent « la trinité ».
} 
Globalement la médiation se définit avant tout comme un processus de communication éthique reposant sur la responsabilité et l'autonomie des participants, dans lequel un tiers impartial, indépendant, neutre, avec la seule autorité que lui reconnaissent les médieurs - favorise par des entretiens confidentiels l'établissement, le rétablissement du lien social, la prévention ou le règlement de la situation en cause.

En tant que «définition globale », elle concerne diverses manifestations du phénomène médiatif. Elle recouvre, d'une part, les médiations de différences et de différends et, d'autre part, elle en ponctue son unité fondamentale. Également, elle s'écarte d'une conception qui ne tient compte que des conflits en épuisant cette unité. D'ailleurs, «l'assimilation de la médiation à une simple technique de règlement non juridictionnel des conflits est la confusion majeure, la plus attentatoire à l'autonomie de la médiation et la plus réductrice de sa nature » (Ibidem : 80).

Bref, deux modèles se confrontent et se concurrencent en fonction de la finalité de la médiation. L'un, le modèle nord-américain, réduit la médiation à un moule à résolution de litiges. L'autre, le modèle français, insiste sur la promotion de la médiation en tant que processus constructeur ou reconstructeur de liens sociaux.

Nous nous interrogeons sur les retombées linguistiques venant de l'adoption d'un modèle au lieu d'un autre. Autrement dit, nous nous demandons dans quelle mesure l'influence du modèle nord-américain a affecté les discours sur la médiation (DE GiOIA \& MARCON, 2014a). Plus précisément, nous nous concentrons sur l'influence lexicale et terminologique que ce modèle a eue dans deux langues, le français et l'italien, et dans trois pays européens, la France, l'Italie et la Belgique.

Pour la France, nous vérifions jusqu'à quel point le modèle des années $80 \mathrm{a}$ réussi à préserver le discours français sur la médiation de l'intégration d'anglicismes, soit d'emprunts, de calques, d'adaptations d'unités lexicales et de termes anglais issus du modèle nord-américain.

Pour l'Italie, nous mesurons la concurrence entre le modèle nord-américain et le modèle français par l'observation de la circulation et de l'implantation éventuelles d'anglicismes dans le discours italien sur la médiation. D'ailleurs, ce discours connaît son essor en Italie avec la prolifération d'ouvrages suivant, d'une part, l'adoption du CECRL (Cadre européen commun de référence pour les langues; VERGNE, 2010) et, d'autre part, la réception de la directive européenne 2008/52/CE (DE GIOIA \& MARCON, 2016). Malgré l'ouvrage de Castelli et d'autres réflexions scientifiques, l'Italie n'a pas réellement contribué à la mise en place d'un modèle de médiation. En raison de ce manque, il est intéressant de mesurer l'emprise de tel ou tel autre modèle.

Pour la Belgique, nous observons de quelle manière le discours belge francophone sur la médiation ressemble aux discours français et italien et s'il est porteur d'anglicismes montrant l'influence du modèle nord-américain.

Pour répondre à ces questions, nous allons vérifier et analyser la présence d'anglicismes dans des sites Web français et italiens concernant la médiation. Ensuite, nous prendrons en compte d'autres anglicismes enregistrés dans notre ouvrage terminographique sur la médiation, qui décrit et met en équivalence des 
termes tirés de deux manuels de médiation civile parus en Italie et en Belgique. Notre but est de donner une vision plus européenne de la médiation.

\section{Un corpus constitué de sites Web et d'un ouvrage terminographique bilingue}

Premièrement, nous prenons en compte environ 40 sites internet de centres de médiation en Italie et en France.

En Italie, il existe un Albo degli Organismi di Mediazione (tableau des centres de médiation), qui est publié sur le site Web du Ministero della Giustizia, Dipartimento per gli affari di giustizia, Direzione della giustizia civile ${ }^{5}$. Dans ce tableau figurent à présent plus de mille centres agréés par le ministère italien ${ }^{6}, \mathrm{y}$ compris ceux dont l'inscription a été ensuite annulée. Leurs sièges n'ont aucun lien avec les institutions juridiques italiennes. Tout au plus, les centres sont enchâssés dans d'autres organismes de nature non juridique, tels que les Camere di commercio.

En France, il existe un annuaire des services juridiques géré par le Ministère de

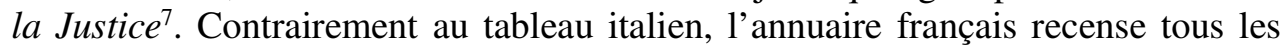
services juridiques. En ce qui concerne la médiation, il se concentre sur des associations, des antennes et des points d'accès au droit dont le siège peut se trouver au sein d'institutions juridiques, par exemple des tribunaux. Nous avons ainsi porté notre attention sur les associations et fédérations des centres de médiation. Nous avons consulté l'Annuaire national des centres de médiation mis au point par la Fédération nationale des centres de médiation (FNCM) en 2014-2015, la liste des centres de médiation disponible sur le site de l'Association nationale des médiateurs (ANM), l'annuaire des centres de médiation disponible sur le site de la Fédération nationale de la médiation et des espaces familiaux (FENAMEF) et la liste des médiateurs du service public sur le site du Club des médiateurs des services au public ${ }^{8}$.

Nous nous concentrons sur l'influence linguistique nord-américaine telle qu'elle apparaît sur les sites internet parce qu'ils représentent l'une des interfaces privilégiées de présentation de la médiation/mediazione au grand public.

\footnotetext{
5 Cf. <https://mediazione.giustizia.it/ROM/ALBOORGANISMIMEDIAZIONE.ASPX> (dernière consultation effectuée le 17 septembre 2018).

${ }^{6}$ Pour les sites italiens, nous avions choisi de prendre en considération les 20 premiers qui se trouvaient inscrits à la date du 15 juin 2016. À cette date, 739 centres étaient affichés au tableau, alors qu'à la date de notre dernière consultation (effectuée le 17 septembre 2018) on en comptait 1077. Nous précisons que l'inscription au tableau est obligatoire. Autrement dit, l'agrément ministériel est indispensable. Cette inscription concerne les centres de médiation en matière civile. Néanmoins, on constate que les centres s'occupent également de médiation dans d'autres matières.

${ }^{7} \mathrm{Cf}$. <http://www.annuaires.justice.gouv.fr/> (dernière consultation effectuée le 17 septembre 2018).

${ }^{8}$ Nous avions consulté les sites Web entre la fin du mois de novembre et le début du mois de décembre 2016. Pour l'annuaire de la FNCM, nous n'avions consulté que les liens explicités et encore actifs pour un total de 10 sites Web. En ce qui concerne le site de l'ANM (section Médiateurs > Liste des organismes), nous avions consulté toute la liste suivant l'ordre alphabétique, retenant 4 sites Web. Du riche annuaire des services de médiation familiale de la FENAMEF, nous avions sélectionné les 3 premiers sites Web disponibles. Pour conclure, dans les sections Médiateurs $>$ Banque, Assurance, Mutuelle et Institutions financières / Téléphonie, Internet et communications électroniques du site Web du Club des médiateurs des services au public, nous avions choisi au hasard 3 autres sites Web.
} 
Autrement dit, les sites des organismes de médiation sont censés présenter à leur public potentiel une définition de la médiation, une explication essentielle sur la nature de la médiation, tout comme les avantages que peut offrir le recours à la médiation.

À partir de ce constat, nous avons établi une grille de description commune pour les deux listes de sites Web reprenant :

- la source du site Web ;

- la dénomination du centre de médiation ;

- la définition de la médiation disponible sur le site Web, ainsi que d'autres remarques portant sur la nature de la médiation ;

- les anglicismes (emprunts, calques, adaptations) éventuellement repérés sur la page d'accueil des sites Web ;

- les anglicismes (emprunts, calques, adaptations) repérés dans des glossaires éventuels mis à la disposition des usagers des sites Web.

Deuxièmement, nous examinons des anglicismes tirés d'un ouvrage terminographique sur la médiation, intitulé Mots de médiation. Un lexique bilingue français-italien / Parole di mediazione. Un lessico bilingue francese-italiano (DE GIOIA \& MARCON, 2014b). Cet ouvrage décrit et met en équivalence des termes extraits de deux publications, italienne et belge, qui sont analogues par leurs contenus, c'est-à-dire des manuels dédiés à l'illustration de méthodes de conduite d'une procédure de médiation civile. Ce qui peut ainsi représenter une autre image européenne de la médiation.

\section{La démarche d'analyse}

Dans les paragraphes qui suivent, nous allons commenter les résultats obtenus dans une perspective comparée.

Entre autres, nous allons mettre en évidence la filiation sémantique de certains emprunts évoquée par DAUZAT (1946), afin de montrer à quel point elle risque de mettre en danger la cohérence et l'autonomie de la médiation.

Dans la ligne de la reconstruction sémantique en diachronie de l'emprunt, envisagée par DEROY (1956), nous allons essayer de mettre en avant l'influence anglophone concernant la mediation, notamment en milieu italophone où le concept mediazione ne bénéficie pas, à notre connaissance, d'une réflexion théorique de référence comme le concept médiation en milieu francophone.

Ensuite, suivant le modèle d'une étude comparée de JACQUET-PFAU, SABLAYROLLES \& HUMBLEY (2011), nous nous focalisons sur les emprunts tout comme sur les processus de calque et de traduction pour l'identification d'autres emprunts «sous influence» dans le domaine de la médiation. Plus précisément, l'observation de la présence ou de l'absence d'équivalents de l'anglais (langue source) par rapport à l'italien et au français (langues cibles) nous permet de mieux apprécier l'influence ou l'indépendance éventuelles de la conceptualisation de mediation proposée en milieu anglophone relativement à la conceptualisation de médiation et de mediazione en milieux francophone et italophone. Nous allons 
également observer comment le recours à l'emprunt, au calque ou à l'adaptation influence le discours autour de médiation et de mediazione respectivement en français et en italien.

En tout cas, notre but n'est pas ici de rendre compte de distinctions théoriques fines entre divers types d'anglicismes, qui peuvent être qualifiés tout à tour comme emprunts, calques, adaptations, créations sous influence, internationalismes etc., d'après diverses optiques linguistiques, mais d'éclairer leur présence afin de vérifier l'influence nord-américaine.

\section{Analyse d'anglicismes tirés de sites Web}

Commençons par la définition et la nature de mediazione / médiation. Aussi bien en italien qu'en français, l'influence du modèle nord-américain a introduit la désignation Alternative Dispute Resolution (et son sigle ADR) par le biais des calques : modes alternatifs de règlement des conflits (MARC) ou des litiges (MARL) ou des différends (MARD) en français-langue cible ; modi alternativi di risoluzione delle controversie en italien-langue cible. Dans les deux langues sœurs, nous observons tout de suite le glissement de la tête nominale de l'anglais resolution vers le choix de modi / modes, dont l'effet est de mettre en relief le caractère alternatif extrajudiciaire.

La quasi-totalité (soit 18 sur 20) des définitions présentes sur les sites Web italiens ramène la médiation à d'autres calques, à savoir uno (strumento + metodo + modo) di (soluzione + risoluzione + composizione) stragiudiziale di (controversie + conflitti), c'est-à-dire à un mode de résolution extrajudiciaire des litiges. C'est bien l'idéologie de la « justice douce », très influencée par les USA, via le Canada, qui s'impose de plus en plus dans les pays de droit continental et en Europe. Six l'appelle la «justice Canada dry »(GUILLAUME-HoFNUNG, 2015 : 115), qu'on retrouve sans nul doute en Italie :

Il Servizio di mediazione offre la possibilità di giungere alla composizione delle controversie civili e commerciali, vertenti su diritti disponibili, tra due o più soggetti comprese le liti tra imprese e tra imprese e consumatori, tramite l'assistenza di un mediatore indipendente, imparziale e neutrale. ${ }^{9}$

Cette définition est donnée tant sur le site de la Camera di Conciliazione della Camera di Commercio Bologna que dans celui du Servizio di conciliazione della Camera di commercio di Crotone, mais elle est analogue aux autres que nous avons repérées. Elle véhicule l'idée d'une autre voie de/ la justice, une voie alternative, certes plus souple et rapide que la justice considérée comme rigoureuse et lente. En

\footnotetext{
9 «Le service de médiation offre la possibilité de parvenir à la composition des litiges civils et commerciaux, portant sur les droits disponibles, entre deux ou plusieurs sujets, y compris les litiges entre entreprises et entre entreprises et consommateurs, grâce à l'aide d'un médiateur indépendant, impartial et neutre $\gg($ Notre traduction).
} 
même temps, elle englobe la médiation dans la justice car, même si c'est une autre voie, elle reste en tout cas en deçà du droit ${ }^{10}$.

En premier lieu, nous remarquons la présence de l'unité lexicale controversia dans la définition citée ci-dessus. Le dictionnaire bilingue grand public ${ }^{11}$ enregistre cette unité dans le domaine du droit et l'indique comme équivalent aussi bien de différend et de conflit que de litige. D'ailleurs, elle est traduisible dans ce contexte par litige. La médiation n'existerait donc pas sans litige, ou en dehors de tout conflit. En revanche, la définition globale proposée par Guillaume-Hofnung nous apprend que la médiation concerne aussi bien les différends que, en amont, les différences et qu'elle vise la communication éthique, non pas le droit.

Pourtant, la controversia est mentionnée dans 18 sites Web sur 20 (notamment dans des textes de définition ou de présentation de la médiation). En outre, l'unité hyperordonnée conflitto est clairement citée dans deux sites. Cette dernière évoque normalement des connotations négatives pour le grand public ${ }^{12}$, alors qu'elle peut impliquer une valeur positive en médiation. Le conflit devient alors le moyen de visualiser l'opposition de plusieurs représentations sociales (Ibidem : 94-95).

En second lieu, nous observons la présence de l'unité lexicale conciliazione dans la dénomination officielle de deux centres de mediazione. Il s'agit d'une confusion terminologique et conceptuelle entre deux MARC maintes fois constatée par les spécialistes et qui a même porté au rattachement de la seconde (mediazione) à la première (conciliazione), par exemple dans le Code de Procédure civile français (DE Giola \& MARCON, 2013, 2014a). Cette confusion renforce l'idée que la juridicisation est sous l'emprise de l'idéologie de la « justice douce ». Il en résulte un emploi spécifique de l'unité mediazione, dans presque tous les textes examinés, qui emprunte essentiellement le sens partial nord-américain de MARC.

Des observations analogues surgissent en ce qui concerne les sites français. 17 sites Web sur 21 donnent des définitions où la médiation est mise en rapport avec les MARC-MARL-MARD ; considérons par exemple le site Alsace Médiation ${ }^{13}$ :

La médiation est un mode de prévention et de règlement amiable des différends. Il s'agit d'un processus structuré par lequel le médiateur invite les personnes concernées à exposer leurs points de vue sur la situation conflictuelle, leurs positions, leurs intérêts et leurs besoins pour les aider à construire la solution la plus adéquate.

La médiation permet aux parties de renouer le fil du dialogue et de trouver leur solution au conflit qui les oppose.

Rapide, économique, confidentielle et non contraignante, la médiation est un choix sans risques, alternative efficace aux procédures judiciaires ou d'arbitrage.

\footnotetext{
${ }^{10}$ La réduction de la médiation à une fonction subordonnée à l'exercice du droit, ainsi que sa juridicisation, passe aussi à travers l'emploi de mots et sens spécifiques du domaine du droit (DE GiOIA \& MARCON, 2013, 2014a). Il y en a plusieurs indices dans la définition de médiation.

${ }_{11}$ Cf. le dictionnaire italien-français en ligne de la série des Dizionari Garzanti Linguistica, $<\mathrm{http}: / /$ www.garzantilinguistica.it/>, à l'entrée controversia.

${ }_{12}$ Voir l'entrée conflit dans le Trésor de la Langue Française, <http://atilf.atilf.fr/>.

$13 \mathrm{Cf}$. <http://alsace-mediation.fr/la-mediation/> (dernière consultation effectuée le 18 septembre 2018).
} 
En outre, la noyade de la médiation dans la justice se produit aussi par les unités litige (dans 5 sites sur 21), différend (11 sur 21), conflit (17 sur 21, ou situation conflictuelle).

Devant l'ensemble des définitions, nous vérifions entre autres l'oscillation entre les unités règlement/régler et solution/solutionner. Même si la première paire sert à ponctuer plutôt la durée du processus et la seconde son aboutissement, le discours textuel sur la médiation est effectivement orienté vers la recherche d'un accord entre les parties qui est très souvent qualifié d'amiable, tout comme d'ailleurs les autres unités règlement et solution.

La juridicisation est observable aussi dans les dénominations des sites Web, où la médiation est associée à d'autres $M A R C$. Mais si dans les dénominations italiennes la médiation s'éclipse devant la conciliation, dans les dénominations françaises elle s'accompagne rarement (3 fois sur 21, ainsi qu'une seule fois dans les définitions) d'un autre $M A R C$, à savoir l'arbitrage (cf. la définition tirée du site français ci-dessus). Ce qui prête à confusion du point de vue conceptuel et terminologique.

Dans les sites Web italiens, les emprunts à l'anglais sont flagrants dans les dénominations mêmes de certains centres. Tout d'abord, des emprunts sémantiques peuvent être reconnus dans l'appellation curieuse de centres de conciliazione alors qu'on s'attendrait à trouver mediazione. Il n'y a pas que les dénominations déjà citées ci-dessus de la Camera di Conciliazione della Camera di Commercio Bologna et du Servizio di conciliazione della Camera di commercio di Crotone. Elles sont analogues à Organismo di conciliazione bancaria, Servizio di Conciliazione della CCIAA di Firenze, Camera Arbitrale e di Conciliazione della Camera di Commercio I.A.A. di Catania, Sportello di Conciliazione della Camera di Commercio I.A.A. di Pisa, Organismo di Conciliazione dell'Ordine degli Avvocati di Monza, Camera Arbitrale e di Conciliazione dei Dottori Commercialisti, A.S.A.C. - Associazione per lo Sviluppo delle Agenzie di Conciliazione, Associazione Nazionale per l'Arbitrato \& la Conciliazione in sigla A.N.P.A.R.

Toutes ces dénominations montrent la filiation sémantique et conceptuelle : conciliazione > mediazione, tout comme son lien avec l'arbitrato. En effet, arbitrato et conciliazione, en particulier, sont deux dispositifs normés en Italie bien avant l'introduction des normes sur la médiation, la toute première remontant à la réception de la directive européenne 2008/52/CE en 2010.

Il est intéressant de constater que cette filiation conciliazione $>$ mediazione - un néologisme sémantique qui subit un changement de sens dès sa parution, surtout en droit -, le deuxième élément se greffant sur le premier, est également soulignée dans le discours du législateur italien, par exemple dans le decreto-legge 21 giugno 2013 n. 69. L'article 1 alinéa c) de ce texte législatif définit la conciliazione comme « la composizione di una controversia a seguito dello svolgimento della mediazione $»^{14}$. D'après cette définition, conciliazione et mediazione se trouvent dans une relation temporelle et causale. Autrement dit, il y a conciliazione quand il y a mediazione

14 « le règlement d'un différend à la suite du déroulement de la médiation » (Notre traduction). 
et/ou parce qu'il y a mediazione, ce qui fait écho à « l'impasse de la médiationconciliation » observée par GUILLAUME-HOFNUNG (2015). L'autonomie des deux concepts est ainsi neutralisée en Italie. Cela est vrai pour le discours du législateur ainsi que pour le discours des centres de médiation italiens.

Pour couronner le tout, parmi les dénominations, on trouve également $A D R$ Concilmed et Concilia Srl, où mediazione s'affilie morphologiquement à conciliazione ou bien disparaît totalement. Or, ce dernier exemple montre non seulement l'emprunt sémantique, toujours d'origine nord-américaine, mais aussi l'emprunt lexical du sigle anglais $A D R$ ramenant la disparition à l'expérience nordaméricaine de l'Alternative Dispute Resolution. Le même emprunt lexical est présent dans les autres dénominations de ADR Notariato s.r.l., Fondazione Aequitas $A D R, A D R$ Center, cette dernière représentant un anglicisme (sémantique et) lexical total, pour ainsi dire ${ }^{15}$.

Dans les sites Web français, comme nous l'avons vu plus haut, l'appellation de médiation entraîne celle d'arbitrage, et ce dans les dénominations de CIMA Centre Interprofessionnel de Médiation et d'Arbitrage, CMAP - Centre de Médiation et d'Arbitrage de Paris, CAMI - Chambre d'Arbitrage et de Médiation des litiges de l'Immobilier.

D'autres emprunts dont l'anglais est la langue source sont repérables dans quelques pages d'accueil des centres de médiation italiens. Outre $A D R$, un cas emblématique est représenté par l'emprunt ombudsman. Une reconstruction de l'évolution sémantique en diachronie de ce terme d'origine scandinave est mise au point par Lozzi Gallo (CoNENNA, 2012 : 182-183). Selon Lozzi Gallo, en suédois et en danois, le terme a le sens de «serviteur du roi, fonctionnaire». Sa resémantisation a lieu au moment où il devient un emprunt du suédois à l'anglais vers la moitié du $\mathrm{XX}^{\mathrm{e}}$ siècle. C'est à cette époque que le terme ombudsman commence à désigner une figure institutionnelle chargée de la défense des droits.

Dans les sites Web italiens, cet emprunt est employé dans les secteurs de l'administration publique et bancaire ${ }^{16}$. Dans le premier cas, le terme ombudsman désigne la figure du «défenseur » des usagers contre la mauvaise administration. Dans le second, le terme Ombudsman bancario identifie l'organe collégial qui règle les différends entre les banques et leurs clients. Ces figures, qui sont censées enquêter sur les plaintes déposées contre des banques (Ombudsman bancario) ou des institutions étatiques (ombudsman) en Italie, remplissent inopinément un vide à la fois administratif/législatif et linguistique.

Pour le premier cas, il s'agit d'une véritable innovation : dans ce cas de figure, ombudsman est un emprunt aussi bien du signifiant (la forme est absente en italien) que du signifié et du référent (la figure qui défend les droits des clients d'une banque). Pour le deuxième cas, nous sommes en présence d'un emprunt utilisé en

\footnotetext{
${ }^{15}$ ADR Center est formé de deux unités lexicales de l'anglais, dont la seconde est la variante américaine actuelle de l'anglais britannique Centre. Voir l'entrée center dans le Cambridge Dictionary, $<$ http://dictionary.cambridge.org/it/>.

16 Voir l'entrée ombudsman dans le vocabulaire de l'italien Treccani en ligne, $<\mathrm{http}: / /$ www.treccani.it/>.
} 
raison de sa « couleur locale ». En effet, la désignation de difensore civico aurait été préférable ou, en tout cas, motivée aussi bien du point de vue législatif que du point de vue linguistique. Malgré le manque d'une loi nationale harmonisatrice et d'une figure nationale, la figure du difensore civico préexistait à l'échelle régionale et urbaine italienne. D'ailleurs, suivant la reconstruction de MANZI $(2004)^{17}$, on comprend à quel point l'histoire des tentatives de création d'un Difensore civico nazionale italien s'est appuyée sur le modèle suédois anglicisé de l'ombudsman ${ }^{18}$. (La France s'y est-elle appuyée elle aussi lorsqu'en 2011 elle remplaça le Médiateur de la République par le Défenseur des droits ${ }^{19}$ ?). Le rôle non neutre joué par la « couleur locale » de l'emprunt ombudsman dans les sites Web italiens paraît ainsi évident dans ce cas.

De plus, ces figures ne doivent pas être confondues avec la figure du médiateur envisagée par la définition globale de Guillaume-Hofnung (cf. plus haut) car elles manquent d'une qualité précise, à savoir l'indépendance. Elles ne sauraient donc être soumises à un pouvoir hiérarchique, ni à une pression politique, financière ou morale, ni à une procédure préconçue.

Dans les sites Web français, nous n'avons trouvé aucun anglicisme lexical dans les pages d'accueil, ni dans les glossaires éventuels mis à la disposition des usagers.

\section{Analyse d'anglicismes tirés d'un ouvrage terminographique bilingue}

Il s'agit de notre volume intitulé Mots de médiation. Un lexique bilingue français-italien / Parole di mediazione. Un lessico bilingue francese-italiano (DE GIOIA \& MARCON, 2014b), qui témoigne aussi du discours belge francophone puisqu'il enregistre principalement des termes tirés de deux manuels de médiation civile parus en Italie et en Belgique. Les anglicismes figurant en entrée dans ce volume sont au nombre de 6 pour l'italien et de 4 pour le français, sur un total de 102 termes pour chaque langue. Même dans le domaine de la médiation, l'italien apparaît comme une langue plus ouverte à l'accueil des anglicismes.

Considérons le début du processus de médiation où le médiateur rencontre les autres acteurs. En français, il leur présentera le cadre de la médiation, soit l'environnement où aura lieu le processus ainsi que la conduite à adopter pour la communication. Il exploitera une liste de questions pour comprendre leurs intérêts et leurs positions. En italien, pour nommer le cadre et la liste de questions, le médiateur aura recours aux emprunts setting et check-list (Ibidem : 94-95, 192-193).

Cependant, ce n'est qu'en français que nous retrouvons un terme résultant d'un calque lexical et sémantique de l'anglais ghost figure. Alors que le français exploite

\footnotetext{
${ }^{17} \mathrm{Au}$ passage, nous remarquons l'utilisation quasi-synonymique d'Ombudsman italiano et de Difensore civico nazionale par Manzi dans son étude.

${ }_{18} \mathrm{Au}$ sein de l'Union européenne, le terme ombudsman est présent dans l'appellation officielle European Ombudsman, dont « la traduction » institutionnelle est Mediatore europeo pour l'italien et Médiateur européen pour le français. En s'appuyant sur cet exemple, ConENNA (2012) a abordé la dissymétrie linguistique et législative en matière de médiation entre Italie et France, tout comme la centralité de l'anglais en droit européen.

${ }^{19} \mathrm{Cf}$. <http://www.defenseurdesdroits.fr/fr > (dernière consultation effectuée le 18 septembre 2018).
} 
la traduction fantôme pour désigner une personne qui influence le processus de médiation de l'extérieur en vertu des relations qu'il entretient avec l'un des acteurs, l'italien emploie le terme referente (Ibidem : 162-163) ${ }^{20}$.

Il y a aussi un cas où l'emploi d'un emprunt à l'anglais américain semble l'emporter sur l'usage des unités équivalentes dans les langues sœurs. Lors d'un processus de médiation, il peut être demandé une forme d'échange entre un médiateur et un des acteurs. Pour y faire référence, bien que les termes sémantiquement équivalents d'entretien confidentiel et de sessione separata soient attestés, il y a une tendance en français comme en italien à emprunter et resémantiser le terme caucus, quoique ce dernier se confonde avec d'autres situations qui n'ont rien à voir avec la médiation, à savoir les réunions électorales états-uniennes. Il s'agit somme toute d'un emprunt à la culture nord-américaine qui n'a pas lieu d'être.

En outre, d'autres emprunts compromettent l'image de la médiation en lui donnant des traits possibles de la négociation. En effet, les deux MARC envisagent un espace commun aux positions et aux intérêts des acteurs que le médiateur et le négociateur reconnaissent ou favorisent pour que les acteurs trouvent un accord. Il en dérive des termes enregistrés en entrées dans notre volume et employés en médiation aussi bien qu'en négociation, en français : zone d'accord possible et son acronyme ZAP, et en italien : zona di possibile accordo et son acronyme ZOPA qui est identique à l'acronyme correspondant de la dénomination anglaise originaire de Zone of Possible Agreement. C'est un acronyme qui est aussi un calque.

À ces termes sont reliés d'autres termes pour lesquels peut être menée une observation analogue à la précédente. En effet, en médiation et en négociation il est possible d'identifier une «meilleure solution de rechange», d'où l'acronyme terminologique français Mesore (avec la variante graphique MESORE en lettres capitales), qui consiste dans l'alternative réaliste que chaque acteur envisage d'accepter en cas d'échec réel ou éventuel du processus de médiation. Comme équivalents sémantiques de l'italien, on trouve l'acronyme Maan et sa variante graphique MAAN pour Migliore Alternativa a un Accordo (Negoziato + Negoziabile + Negoziale), qui équivaut à l'anglais BATNA: Best Alternative to a Negotiated Agreement. Ici aussi, on a affaire à un acronyme qui est un calque. Remarquons l'existence d'antonymes en italien et en anglais, mais pas en français : PAAN: Peggiore Alternativa a un Accordo (Negoziato + Negoziabile + Negoziale) / WATNA : Worst Alternative to a Negotiated Agreement.

Enfin, dans notre Lexique on découvre aussi trois anglicismes ne figurant pas en entrée mais dans d'autres sections des fiches terminographiques. Ce sont les emprunts Alternative Dispute Resolution, ombudsman, win-win, qui confirment une fois de plus l'anamorphose de la médiation qui lui est infligée par le droit (Guillaume-Hofnung, 2016). Nous avons déjà examiné plus haut les deux premiers anglicismes, mais le troisième est aussi emblématique. Dans les pays anglo-saxons où la médiation est vue comme un MARC finalisé à trouver un accord, et non pas à encourager la communication éthique, la médiation est dite win-win seulement si elle parvient à un accord qui satisfait les deux parties.

${ }^{20} \mathrm{C}$ 'est aussi un exemple de matrice interne $>$ changement de sens $>$ extension du sens. 


\section{Conclusion}

En France, en Italie et en Belgique, l'existence d'emprunts à l'univers nordaméricain est évidente en médiation. Ces emprunts sont essentiellement de deux types car on emprunte aussi bien le modèle nord-américain de la médiation que ses unités lexicales et terminologiques, que l'on peut généralement qualifier d'anglicismes. Ce sont les deux facettes d'une même médaille.

En France comme en Belgique coexistent deux modèles de médiation, l'un nordaméricain et l'autre français, expression du droit civil, européen de par sa nature. Ce modèle résiste encore au pattern nord-américain envahissant, grâce aussi, nous semble-t-il, à la tradition de réflexions théoriques et de pratiques de terrain en cours depuis les années 1980. En Italie, où il n'existe pas une tradition de ce type, le modèle nord-américain bat son plein.

La « couleur locale » de l'anglicisme (que ce soit emprunt, calque, adaptation etc.) porte atteinte à l'unité conceptuelle de la médiation. Elle transfère une «couleur exotique » dans la mesure où elle transplante une conceptualisation externe inconnue en langue cible. Autrement dit, la vision nord-américaine de la médiation en tant qu'ADR influence le modèle francophone préexistant et « colonise » d'autres territoires linguistiques, comme l'Italie et l'italien, dépourvus d'un modèle théorique de mediazione préexistant.

À l'égard de l'influence sur un modèle préexistant, comme il se passe en France, la «couleur locale » de l'anglicisme non seulement entrave la compréhension des locuteurs moyens de la langue cible (de quelle médiation parle-t-on ?), mais aussi elle contribue à la fragmentation du concept de médiation, tout comme à la déstabilisation et à la menace du modèle de médiation préexistant.

Dans le cas de figure où la «couleur locale » de l'anglicisme s'instaure dans une langue cible dépourvue d'un modèle de médiation, comme cela se passe en Italie, on assiste à une internationalisation du modèle nord-américain et, par conséquent, au passage des termes au statut d'internationalismes. Dans cette perspective, nous sommes loin de la conception de la médiation comme un «avatar vernaculaire [...] d'une idée commune à de nombreuses cultures » (GUILLAUMEHoFNUNG, 2015: 11). Au contraire, nous sommes en présence d'une action d'exportation-importation d'un modèle de médiation qui empêche le développement éventuel d'un modèle autochtone. De plus, cette action d'exportation-importation peut conduire à des aberrations, comme le représente l'introduction, en 2013, du caractère obligatoire de la médiation pour la résolution de certains conflits avant tout recours à la voie judiciaire en Italie. Le caractère obligatoire est en soi contraire au caractère volontaire intrinsèque au processus de la médiation dans toutes ses pratiques.

La présente étude se veut une démonstration de cette exportation-importation, fondée sur l'analyse conceptuelle et linguistique de plusieurs anglicismes tirés d'un choix de sites Web français et italiens, ainsi que de notre volume Mots de médiation. Un lexique bilingue français-italien / Parole di mediazione. Un lessico bilingue francese-italiano (DE GIOIA \& MARCON, 2014b).

Par ailleurs, l'analyse conceptuelle et terminologique que nous avons menée peut contribuer à la construction d'une ontoterminologie de la médiation. 
L'évaluation différentielle des sens des concepts et des termes peut servir à mieux les cerner, à distinguer les définitions formelles des concepts (spécifications logiques) des définitions en langue naturelle des termes (explications linguistiques), à étiqueter les données linguistiques non plus par des mots-clés mais par des concepts en les reliant selon leurs rapports hiérarchiques.

\section{BIBLIOGRAPHIE}

BONAFÉ-SCHMITT J.-P. (2013), «Les modèles de médiation: Modèles latins et anglo-saxons de médiation », in: Nicolau G. (dir), La médiation, entre renouvellement de l'offre de justice et droit, Jurisprudence - Revue critique, 4, Université de Savoie, p. 151-169.

CASTELli S. (1996), La mediazione. Teorie e tecniche, Milan, Raffaello Cortina.

ConenNA M. (2012), « Médiation / Traduction », in : Lautel-Ribstein F. \& Masson J.-Y. (éd), Jean-René Ladmiral : une æuvre en mouvement. Revue SEPTET Des mots aux actes, 3, Perros-Guirec, Anagrammes, p. 176-189.

DAUZAT A. (1946 [1945]), « Les mots d'emprunt dans l'argot français », in : Études de linguistique française, Paris, D’Artrey, p. 262-305.

De Gioia M., MARCON M. (2013), « Médiation civile et terminologie juridique. Un regard croisé français-italien », in : De Gioia M. (dir), Autour de la traduction juridique, Padoue, Padova University Press, p. 17-46.

De Gioia M., MARCon M. (2014a), «Discours de médiation(s). Le cas de 'conflit' / 'conflitto' », in : De Gioia M. (éd), Pratiques communicatives de la médiation. Actes du Colloque international (Université de Padoue, 6-7 décembre 2012), Berne, Peter Lang, p. 39-71.

De Giola M., Marcon M. (2014b), Mots de médiation. Un lexique bilingue français-italien / Parole di mediazione. Un lessico bilingue francese-italiano, préface de M. Guillaume-Hofnung, Padoue, Padova University Press.

DE GioIA M., MARCON M. (2016), « Remarques terminologiques sur la directive 2008/52/CE du Parlement européen et du Conseil », in : De Gioia M., Marcon M., (dir), Approches linguistiques de la médiation, Limoges, Lambert-Lucas, p. 11-29.

DEROY L. (1956), L'emprunt linguistique, Paris, Société d'édition «Les Belles Lettres $»$.

FISHER R., URY W., PATTON B. (1982), Getting to yes: negotiating agreement without giving in, $1^{\text {st }}$ ed., London, Hutchinson.

Guillaume-Hofnung M. (2015 [1995]), La Médiation, 7éd. mise à jour, Paris, Puf (coll. «Que-sais-je ? », 2930).

Guillaume-HofNung M. (2016), «Pour un statut épistémologique garant de la médiation », in : De Gioia M., Agresti G., Marcon M. (dir), Médiation et droits linguistiques. Actes du Colloque international (Université de Padoue, 23 janvier 2014), Études de Linguistique Appliquée, 181, janvier-mars 2016, Paris, Klincksieck / Didier Érudition, p. 23-34.

Humbley J. (2017), «La néologie de la médiation », in : De Gioia M., GourvèsHayward A., Sablé C. (éd), Acteurs et formes de médiation pour le dialogue 
interculturel. GLAT Padova 2016. Actes du Colloque international (Université de Padoue, 17-19 mai 2016), Brest, Télécom Bretagne, Institut Mines-Télécom, p. 135-144.

JacQuet-Pfau C., Sablayrolles J.-F., Humbley J. (2011), «Emprunts, créations 'sous influence' et équivalents », in : Van Campenhoudt M., Lino T., Costa R. (dir), Passeurs de mots, passeurs d'espoir : lexicologie, terminologie et traduction face au défi de la diversité. Actes des Huitièmes Journées scientifiques du Réseau de chercheurs 'Lexicologie, terminologie, traduction'. Lisbonne, 15-17 octobre 2009, Édition des archives contemporaines et Agence universitaire de la Francophonie, p. 325-339.

LinN A. R. (2011), «Impact: Linguistics in the Real World», in : Linn A., Candel D., Léon J. (dir), Linguistique appliquée et disciplinarisation, Histoire Epistémologie Langage, 33:1, Paris, SHESL, p. 15-27.

MANZI I. (2004), «Il Difensore civico: storia e prospettive dell'Ombudsman italiano », Diritto \& Diritti, <http://www.diritto.it/sentenze/tarmarche/ manzi.html $>$ (dernier accès effectué le 18 septembre 2018).

SIX J.-F. (1990), Le temps des médiateurs, Paris, Seuil.

VERGNE M. (2010), «La situation de médiation dans l'apprentissage d'une langue », Lingua viva, 10, p. 15-30. 